Proceedings

\title{
Expression of Apoptotic Proteins Bax and Bcl-2 in Blood Cells of Type 2 Diabetic Patients ${ }^{\dagger}$
}

\author{
Ebru Gokalp-Ozkorkmaz 1,*, Günsel Kirman ${ }^{1}$, Zafer Pekkolay ${ }^{2}$, Firat Asir ${ }^{1}$ and \\ Engin Deveci ${ }^{1}$ \\ 1 Department of Histology and Embryology, Faculty of Medicine, Dicle University, 21100 Diyarbakır, \\ Turkey; gkirman21@gmail.com (G.K.); firatasir@gmail.com (F.A.); engindeveci64@gmail.com (E.D.) \\ 2 Department of Endocrinology, Faculty of Medicine, Dicle University, 21100 Diyarbakır, Turkey; \\ drpekkolay@gmail.com \\ * Correspondence: ebrug76@gmail.com \\ + Presented at the 2nd International Cell Death Research Congress, Izmir, Turkey, 1-4 November 2018. \\ Published: 10 December 2018
}

\begin{abstract}
Diabetes mellitus (DM) is a complex disease characterized by absolute insulin deficiency or resistance leading to hyperglycemia. Type $2 \mathrm{DM}$ is caused by development of cellular resistance to insulin combined with insufficient insulin production. Type $2 \mathrm{DM}$ is a common disorder causing hyperglycemia, impaired homeostatic process, inhibition of inflammatory response, generation of reactive oxygen species. Apoptosis is a crucial process that influence normal development and tissue homeostasis. Apoptosis is known to be reduced in diabetics. Apoptotic proteins such as bcl2 and Bax proteins regulate apoptosis. Bcl-2 serves as anti-apoptotic protein while Bax is proapoptotic. The balance between these apoptotic proteins determine the apoptotic process. The aim of this study was to examine the expression of apoptotic proteins Bax and Bcl-2 with immunohistochemical methods in the blood samples of type 2 diabetic patients.
\end{abstract}

Keywords: apoptosis; Bcl-2; Bax; type-2 diabetes; blood cells

\section{Introduction}

Complications associated to diabetes is a serious health issue. Predisposition to apoptosis in diabetics was carried out in previous studies [1], apoptosis involves a group of proteins such as Bcl2 and Bax that serve in the regulation of apoptosis and act to promote or suppress cell death. Apoptosis is a crucial process that influences normal development and tissue homeostasis. This mechanism is quite conserved through evolution and there are tens of millions of cells that undergo this phenomena. Bcl-2 family proteins monitor apoptosis and regulate this complex molecular network. Many cellular events such as DNA damage, energy stress, loss of growth factor signaling and hypoxia can initiate apoptosis by activation of these proteins. The Bcl-2 family proteins takes prominent role and regulation of mitochondrial or intrinsic apoptotic response. Recently, antiapoptotic Bcl-2 family proteins become literaturally popular to develop novel drugs for many diseases. An overexpression of Bax promotes cell death while the overexpression of Bcl-2 enhances cell survival by suppressing apoptosis [2]. Previous studies on blood of diabetics showed the impairment of phagocytic and microbicidal activities of neutrophils and predispose the patient to infection [3]. The aim of this study was to find out the relationship between expression of apoptotic proteins (Bcl-2 and Bax) and blood cells of Type 2 diabetic patients. 


\section{Experimental Procedure}

\subsection{Samples}

Blood samples of control group (n: 20) and patients with Type 2 Diabetes (n: 30) were collected from Dicle University Hospitals. All experimental protocols were approved by the Human Research Ethics Committee of Dicle University and consent forms were obtained from patients.

\subsection{Immunohistochemistry}

The blood fluid was subjected to high-speed centrifugation, the supernatant was discarded, and $10 \%(v / v)$ neutral formalin was added (pellet: formalin volume ratio, 1:3). After $2 \mathrm{~h}$ of fixation, the clear bright supernatant was discarded and the tube was inverted on filter paper to allow excess fluid to drain. The pellet was then placed on filter paper using a spatula and eosin stain solution was added using a Pasteur pipette. After the cell aggregate developed a red color, it was wrapped in filter paper and placed in a cassette, which was then stored in a fixation box for tissue processing. The cells were examined following Harris hematoxylin and eosin staining. Following the routine paraffin protocol, 4-6 $\mu \mathrm{m}$ paraffin sections were cut with a microtome (Leica, Germany). Antigen retrieval process was performed in citrate buffer solution ( $\mathrm{pH}: 6.0$ ) two times ( $5 \mathrm{~min}$ and $3 \mathrm{~min}$, distinctly) in microwave oven at $700 \mathrm{~W}$. The sections were allowed to cool at room temperature for $20 \mathrm{~min}$ and washed in distilled two times water for $4 \mathrm{~min}$. Endogenous peroxidase activity was blocked in 10\% Hydrogen peroxide solution for 7 min. Ultra V block (Histostain-Plus Kit, 1754084A, Novex Life Tech., 7335 Executive Way, Frederick, MD, USA) was applied for 8 min prior to the application of primary antibodies (Bax Antibody Kit, sc-7480, Santa Cruz Biotech, Dallas, TX, USA), (Bcl-2 antibody, Santa Cruz Biotech., sc-509, USA) for overnight. Secondary antibody (Histostain-Plus Kit, 1754084A, Novex Life Tech., Waltham, MA, USA) was applied for $20 \mathrm{~min}$. The sections were then exposed to streptavidin-peroxidase for 20 min. Diaminobenzidine (DAB-Plus Substrate Kit, 1636518A, Novex Life Tech., Waltham, MA, USA) was used as a chromogen. Control slides were prepared as mentioned above but omitting only the primary antibodies step. After counterstaining with Hematoxylin, washing in tap water for $3 \mathrm{~min}$ and in distilled water for $2 \times 3 \mathrm{~min}$, the slides were mounted. Sections were examined under light microscope (Carl Zeiss, Imager A2, Oberkochen, Germany).

\section{Results}

In blood tissue samples of Type 2 diabetic patients, separation of chromatin bridges was seen in granular leukocyte cell nuclei, and positive Bax expression was observed thus, apoptosis was observed in lymphocytes and neutrophil cells. Vacuolar structures, pyknotic and apoptotic changes in the nuclei of some leukocyte cells were observed. A weak Bcl-2 expression was observed in lymphocytes and neutrophil cells in Type 2 diabetic samples (Figures 1 and 2). Control group showed negative expression (Figure 3).

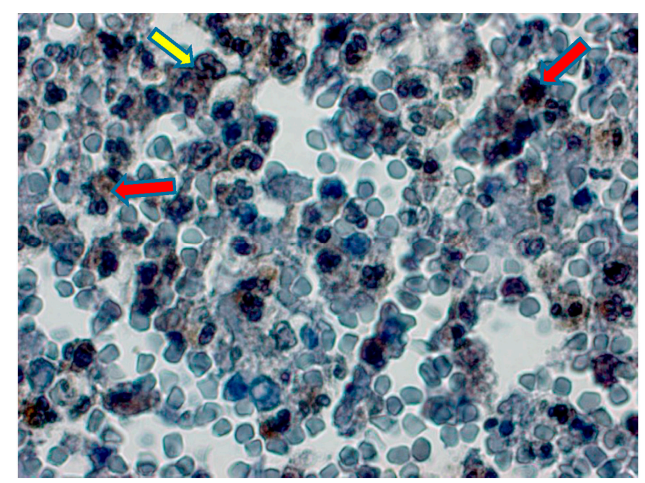

Figure 1. Type 2 diabetic group, separation of chromatin bridges in nuclei of granular leukocyte cells (yellow arrow), degenerative changes and apoptosis in some lymphocyte and neutrophils (red 
arrow). Positive Bax expression in lymphocyte and neutrophil cells. Bax immunochemistry staining, bar $50 \mu \mathrm{m}$.

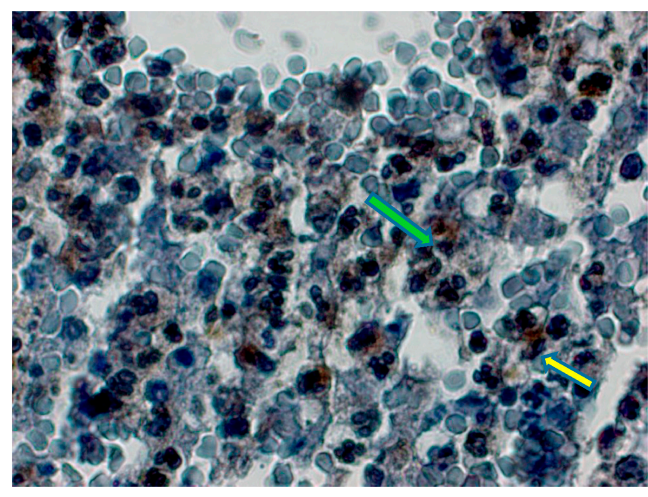

Figure 2. Type 2 diabetic group, vacuolar structures in some leukocyte cells, moderate Bcl-2 expression (yellow arrow), pyknosis in nuclei were observed. Bcl-2 expression and apoptotic changes (green arrow) in nuclei of some lymphocytes. Bcl-2 immunochemistry staining, bar $50 \mu \mathrm{m}$.

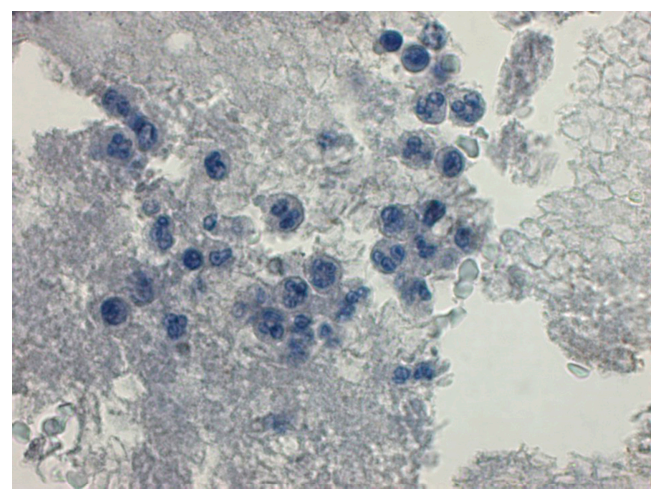

Figure 3. Control group, negative Bax expression was observed in lymphocytes, monocytes and neutrophils. Negative immunochemistry staining, bar $50 \mu \mathrm{m}$.

\section{Discussion}

It is a well-known fact that prolonged hyperglycemia induces dysfunction of several organ systems. Additionally, Type $2 \mathrm{DM}$ is associated with complications in the kidneys, arteries, and eyes and impairment of these are involved in inflammatory processes. There are several studies which demonstrate overexpression of Bcl-2 and Bax proteins in different tissues with hyperglycemia [4].

In a study by Hasnan et al. reported that pro-apoptotic Bax was increased in the blood vessels of diabetic patients [5]. Galkowska et al. also demonstrated that apoptosis in the endothelial cells of diabetic ulcers and overexpression of Bcl-2 [6]. Investigations on blood tissue of diabetic patients confirmed impaired chemotactic, phagocytic and microbicidal activities of neutrophils. Reduced release of cytokines and prostaglandin by neutrophils, increased leukocyte apoptosis are other alterations occur in diabetes [7]. Hyperglycemia decreases phagocytic activity of leukocytes in diabetic patients is known by the researchers. These may be due to the combination of a series of metabolic pathways including free radical formation and improper apoptosis in blood cells of diabetics. Xia et al. revealed that, T lymphocyte plays an important role in the pathogenesis of Type $2 \mathrm{DM}$ as because these cells have regulatory effects on especially chronic inflammation [8]. Our observations indicated that apoptotic changes in the cytoplasm and nuclei of lymphocytes and neutrophil cells and also, pyknosis in these cell nuclei were observed in Type-2 diabetic samples. Bax expression seemed to be more prominent than Bcl-2 expression. Immunostaining results we obtained from the present study revealed that the apoptotic process is in favor of Bax expression. 
Author Contributions: E.G.O. designed the experiments and wrote the paper, E.D. designed the experiments and analyzed the data, Z.P. diagnosed the diabetic patients, G.K. collected the blood samples and F.A. performed the experiments.

Conflicts of Interest: The authors declare no conflict of interest.

\section{References}

1. Hata, A.N.; Engelman, J.A.; Faber, A.C. The Bcl-2 family: Key mediators of the apoptotic response to targeted anti-cancer therapeutics. Cancer Discov. 2015, 5, 475-487, doi:10.1158/2159-8290.CD-15-0011.

2. Reed, J.C. Bcl-2 and the regulation of programmed cell death. J. Cell Biol. 1994, 124, 1-6.

3. Bagdade, J.D.; Nielson, K.L.; Bulger, R.J. Reversible abnormalities in phagocytic function in poorly controlled diabetic patients. Am. J. Med. Sci. 1972, 263, 451-456.

4. Abu-El-Asrar, A.M.; Dralands, L.; Missotten, L.; Al-Jadaan, I.A.; Geboes, K. Expression of apoptosis markers in the retinas of human subjects with diabetes. Investig. Ophthalmol. Vis. Sci. 2004, 45, 2760-2766, doi:10.1167/iovs.03-1392

5. Hasnan, J.; Yusof, M.I.; Damitri, T.D.; Faridah, A.R.; Adenan, A.S.; Norbaini, T.H. Relationship between apoptotic markers (Bax and Bcl-2) and biochemical markers in type 2 diabetes mellitus. Singap. Med. J. 2010, $51,50$.

6. Galkowska, H.; Olszewsk, W.L.; Wojewodzka, U.; Mijal, J.; Filipiuk, E. Expression of apoptosis- and cell cycle-related proteins in epidermis of venous leg and diabetic foot ulcers. Surgery 2003, 134, 213-220, doi:10.1067/msy.2003.223.

7. Alba-Loureiro, T.C.; Munhoz, C.D.; Martins, J.O.; Cerchiaro, G.A.; Scavone, C.; Curi, R.; Sannomiya, P. Neutrophil function and metabolism in individuals with diabetes mellitus. Braz. J. Med. Biol. Res. 2007, 40, 1037-1044, doi:10.1590/S0100-879X2006005000143.

8. Xia, C.; Rao, X.; Zhong, J. Role of T Lymphocytes in Type 2 Diabetes and Diabetes-Associated Inflammation. J. Diabetes Res. 2017, 6494795, doi:10.1155/2017/6494795.

(C) 2018 by the authors. Licensee MDPI, Basel, Switzerland. This article is an open access article distributed under the terms and conditions of the Creative Commons Attribution (CC BY) license (http://creativecommons.org/licenses/by/4.0/). 